\title{
Amplification lags nonlinearity in the recovery from reduced endocochlear potential
}

\author{
${ }_{5}$ C. Elliott Strimbu ${ }^{1}$, Yi Wang $^{2}$, and Elizabeth S. Olson ${ }^{1,2, *}$ \\ ${ }_{6}^{1}{ }^{1}$ Columbia University Medical Center, Department of Otolaryngology, P\&S 11-452, West 168th Street, New York, NY 10032 \\ $7{ }^{2}$ Columbia University Department of Biomedical Engineering, 1210 Amsterdam Ave., New York, NY 10027 \\ 8 *Correspondence: eao2004@columbia.edu
}

${ }_{9}$ ABSTRACT The mammalian hearing organ, the cochlea, contains an active amplifier to boost the vibrational response to low 10 level sounds. Hallmarks of this active process are sharp location-dependent frequency tuning and compressive nonlinearity over 11 a wide stimulus range. The amplifier relies on outer hair cell $(\mathrm{OHC})$ generated forces driven in part by the endocochlear potential $12(E P)$, the $\sim+80 \mathrm{mV}$ potential maintained in scala media, generated by the stria vascularis. We transiently eliminated the EP in 13 vivo by an intravenous injection of furosemide and measured the vibrations of different layers in the cochlea's organ of Corti 14 using optical coherence tomography. Distortion product otoacoustic emissions (DPOAE) were monitored at the same times. ${ }_{15}$ Following the injection, the vibrations of the basilar membrane lost the best frequency (BF) peak and showed broad tuning similar ${ }_{16}$ to a passive cochlea. The intra-organ of Corti vibrations measured in the region of the OHCs lost their BF peak and showed ${ }_{17}$ low-pass responses, but retained nonlinearity, indicating that $\mathrm{OHC}$ electromotility was still operational. Thus, while electromotility 18 is presumably necessary for amplification, its presence is not sufficient for amplification. The BF peak recovered nearly fully ${ }_{19}$ within 2 hours, along with a non-monotonic DPOAE recovery that suggests that physical shifts in operating condition are a final ${ }_{20}$ step in the recovery process.

SIGNIFICANCE The endocochlear potential, the $+80 \mathrm{mV}$ potential difference across the fluid filled compartments of the cochlea, is essential for normal mechanoelectrical transduction, which leads to receptor potentials in the sensory hair cells when they vibrate in response to sound. Intracochlear vibrations are boosted tremendously by an active nonlinear feedback process that endows the cochlea with its healthy sensitivity and frequency resolution. When the endocochlear potential was reduced by an injection of furosemide, the basilar membrane vibrations resembled those of a passive cochlea, with broad tuning and linear scaling. The vibrations in the region of the outer hair cells also lost the tuned peak, but retained nonlinearity at frequencies below the peak, and these sub-BF responses recovered fairly rapidly. Vibration responses at the peak recovered nearly fully over 2 hours. The staged vibration recovery and a similarly staged DPOAE recovery suggests that physical shifts in operating condition are a final step in the process of cochlear recovery.

\section{${ }_{21}$ INTRODUCTION}

${ }_{22}$ In the mammalian cochlea, the sound signal in the form of traveling pressure+motion waves, is converted to electrical signals by ${ }_{23}$ the sensory hair cells that lie within the the organ of Corti (Fig.1). Arrays of tightly-packed stereocilia (hair bundles) of graded 24 height protrude from the apical surface of the hair cells. During hearing, relative motion between the tectorial membrane, an ${ }_{25}$ acellular structure that overlies and couples the hair cells, and the reticular lamina located at the apical surface of the hair cell ${ }_{26}$ bodies, results in shearing of the hair bundles. When the stereocilia deflect towards/away from their tallest row, mechanically ${ }_{27}$ gated ion channels on the shorter rows are opened/closed and the flow of cations, mostly $\mathrm{K}^{+}$, also $\mathrm{Ca}^{2+}$, is increased/reduced ${ }_{28}$ and depolarizes/hyperpolarizes the hair cells. Complex sounds are resolved into individual frequency components which peak ${ }_{29}$ at different locations along the length of the cochlea with high frequencies encoded at the base and low frequencies encoded at so the apex.

${ }_{31}$ The mechanical vibrations of the organ of Corti Complex (OCC $=$ the organ of Corti and tectorial and basilar membranes, ${ }_{32} \mathrm{TM}$ and $\mathrm{BM}$ ) are boosted by the cochlear amplifier, an active process or set of coupled processes operating under feedback. A зз key component of amplification is generated by the outer hair cells (OHCs): in response to changes in the membrane potential, ${ }_{34}$ the $\mathrm{OHC}$ soma changes length in a process driven by the motor protein prestin (1). The cochlear amplifier increases the response ${ }_{35}$ to low level sounds, resulting in a compressive nonlinearity that boosts the dynamic range of hearing across some six orders 


\section{Strimbu et al.}

${ }_{36}$ of stimulus pressure magnitude (2-4). In healthy cochleae, the vibrations at low sound pressure levels are sharply peaked at ${ }_{37}$ each longitudinal location's best frequency (BF) (Fig. 1C and D). Thus the cochlear amplifier increases both sensitivity and ${ }_{38}$ frequency resolution. In cochleae for which the active process is not functional, for example: post mortem, following mechanical з9 damage, or pharmacological inhibition, the responses become linear and exhibit broad tuning. Broad tuning is also typically ${ }_{40}$ observed at stimulus levels greater than about $80 \mathrm{~dB}$ SPL. OHC electromotility is most likely responsible for amplifying the ${ }_{41}$ OCC vibrations, while the nonlinearity is due to saturation in mechano-electric transduction (MET) (Fig. 1E and F) $(5,6)$. The ${ }_{42}$ nonlinearity of the cochlear amplifier produces distortion product otoacoustic emissions (DPOAEs). When two or more tones ${ }_{43}$ are presented simultaneously, additional frequencies are generated in the cochlea and reverse propagate to the middle ear. The ${ }_{44}$ resulting motion of the eardrum then produces faint sounds at these distortion frequencies that can be measured in the ear canal. ${ }_{45}$ DPOAEs are used as a non-invasive gauge of cochlear condition, and have also been used to detect operating point shifts in the ${ }_{46}$ MET nonlinearity (Fig. 1E) (7, 8).

${ }_{47} \quad$ The MET currents are driven in part by the endocochlear potential (EP), the $\sim+80 \mathrm{mV}$ electrical potential within the ${ }_{48}$ scala media. The EP is generated by cells within the stria vascularis (Fig. 1B). Gradual degradation of the stria vascularis and ${ }_{49}$ the corresponding decline in the EP is one cause of presbyacusis, or age-related hearing loss, which affects up to half of the 50 population over 75 years of age in the United States (9-11). Loop diuretics such as furosemide cause a sudden and reversible ${ }_{51}$ decrease in the EP and have been used in vivo in animal studies to investigate the effects of EP reduction on mechanical (12) ${ }_{52}$ and electrical (13-15) responses within the cochlea, and also on DPOAEs (15-17).

In recent studies by our group (15), furosemide was administered intravenously (iv) to gerbil, and EP was monitored ${ }_{54}$ continuously along with the extracellular voltage measured close to the BM. We termed this voltage "local cochlear microphonic" ${ }_{55}$ (LCM). The LCM shows tuning and traveling wave phase excursion similar to the adjacent BM motion, and is useful for ${ }_{56}$ exploring cochlear amplification (18-20). LCM is a measure of OHC current and its saturation can be used as an in-vivo probe ${ }_{57}$ of saturation and operating point shifts in MET (Fig. 1 E) (15, 20). In the furosemide + LCM experiments, following a deep ${ }_{58}$ reduction in EP and LCM responses, both recovered but with different time scales. Interestingly, LCM could recover fully with ${ }_{59}$ EP still substantially sub-normal, contrary to modeling predictions (21). These points are illustrated in Fig. 2, which shows so time-variation following iv-furosemide of: (A) EP for five preparations; (B) examples of LCM at the BF for two preparations; ${ }_{61}$ (C) MET operating point. Fig. 2A shows that following iv-furosemide, EP recovered and stabilized at a sub-normal voltage by $62 ~ 40$ mins; we assume that a similar time course holds in the current study, which used an identical iv-furosemide protocol. (For ${ }_{63}$ technical/practical reasons, EP could not be simultaneously measured in the current experiments.) In the LCM-recovery data of ${ }_{64}$ expt. 696 (data with crosses in Fig. 2B) at 40 mins LCM was in an early phase of recovery. There was a boost of recovery at $\sim 70$ 65 mins and full recovery at $100 \mathrm{mins}$, even though EP had stabilized at only $60 \mathrm{mV}$ (expt. 696 in panel A). In the LCM-recovery 66 data of expt. 705 (solid line data in Fig. 2B) at $40 \mathrm{mins}$ LCM had recovered somewhat and nearly plateaued, then had a boost of 67 recovery at 50-80 mins. By analyzing the fundamental and harmonics in expt. 705, MET operating point was determined and ${ }_{68}$ its time dependence is shown in Fig. 2C. The study concluded that the delayed LCM recovery coincided with an operating point ${ }_{69}$ shift in MET. To summarize the findings of the LCM studies: The recovery of cochlear amplification occurred many mins after 70 the EP had stabilized at a sub-normal level, and that recovery was concurrent with a recentering of MET operating point.

$71 \quad$ Following that background, in the present study we used phase sensitive spectral domain optical coherence tomography 72 (SD-OCT) (22) to measure the sound-induced vibrations within the OCC in vivo before and after the EP was perturbed by 73 an iv injection of furosemide. Unlike single-point interferometry which only yields a vibration measurement at one position 74 in the optical path, typically the BM when recording from basal locations in the cochlea (2), OCT allows for simultaneous

75 measurements at multiple axial locations. This enables simultaneous recording from different layers within the organ of Corti, 76 and the ability to contrast the motion at the BM and OHC-regions. To give a brief preview of findings: BM vibrations lost their ${ }_{77} \mathrm{BF}$ peak and became nearly passive, similar to findings in previous studies (12). The vibrations in the OHC region lost the ${ }_{78} \mathrm{BF}$ peak and became low-pass, but retained sub-BF compressive nonlinearity. This vibration nonlinearity is taken to be an 79 expression of OHC-electromotility, an expectation that is supported by the observation that the LCM, representing OHC current so (and thus voltage), shows substantial sub-BF nonlinearity when elicited with the multi-tone stimuli used in the present study ${ }_{81}$ (20). The DPOAEs recovered nonmonotonically, with a time course that reinforced the correlation of MET operating point to ${ }_{82}$ the recovery of amplification. Thus, the present findings indicate that (1) the presence of even robust OHC electromotility is 83 not sufficient for effective amplification and (2) effective amplification returns along with a recovery of MET operating point. ${ }_{84}$ These findings advance our understanding of the constellation of factors that sustain the cochlear amplifier and produce the ${ }_{85}$ remarkable sensitivity and frequency resolution of the cochlea.

\section{${ }_{86}$ MATERIALS AND METHODS}

${ }_{87}$ The experiments were approved by the Columbia University Institutional Animal Care and Use Committee. 


\section{${ }_{88}$ Gerbil Preparation}

s9 Young, mature gerbils of either sex were anesthetized with intraperitoneal (IP) injections of ketamine (3-6 mg) and sodium ${ }_{90}$ pentobarbital $(40 \mathrm{mg} / \mathrm{kg})$. Anesthesia was maintained with supplemental doses of pentobarbital given if the animals displayed a ${ }_{91}$ reflex in response to a light toe pinch. Buprenorphone $(0.2 \mathrm{mg} / \mathrm{kg})$ was administered IP every six hours. The gerbil's scalp was ${ }_{92}$ removed and the head attached to a two-axis goniometer (Melles-Griot) with dental cement (Durelon, 3M). The left pinna and ${ }_{93}$ most of the cartilaginous ear canal were resected and the animals were tracheostomized to facilitate breathing. The tissue and ${ }_{94}$ muscle over the left temporal bone were carefully dissected and a narrow opening in the bulla was made by chipping the bone ${ }_{95}$ with fine forceps. A bridge of dental cement was used to firmly attach the bulla to the goniometer. Throughout the surgery and ${ }_{96}$ experiment, the animal's temperature was maintained at $38^{\circ} \mathrm{C}$ with a servo-controlled heating blanket and monitored with ${ }_{97}$ a rectal thermometer. During the OCT measurements, additional heating to the animal's head was supplied by a disposable ${ }_{98}$ hand warmer (Hot Hands, HeatMax Inc.) positioned on the goniometer. Experiments were conducted on an optics table in an 99 acoustical isolation booth (Industrial Acoustics Corp.).

\section{${ }_{100}$ Acoustical System}

${ }_{101}$ Acoustic signals were generated using a Tucker Davis Technologies (TDT) system with a sampling rate of 97656.25 S/s. The ${ }_{102}$ sound was played by a Radio Shack speaker and delivered closed-field to the ear canal through a plastic tube. Pressures were ${ }_{103}$ measured with an ultrasonic microphone (Sokolich) whose probe tube was positioned $1-2 \mathrm{~mm}$ from the tympanic membrane. ${ }_{104}$ Sound pressure levels are reported as $\mathrm{dB}$ SPL referenced to the standard value, $0 \mathrm{~dB}=20 \mu \mathrm{Pa}$. The OCT and TDT systems 105 were synchronized as previously described (22). The clock signal from the TDT zBus was modified using a custom built ${ }_{106}$ digital/analog circuit to give a high duty cycle $(90 \%$ high, $10 \%$ low) $5 \mathrm{~V}$ square wave which served as the TTL trigger for the ${ }_{107}$ OCT's line camera. Tuning curves were measured in response to zwuis tone complexes $(23,24)$; 60 frequencies from $\sim 4 \mathrm{kHz}$ to ${ }_{108} \sim 37 \mathrm{kHz}$ were presented simultaneously for $2^{20}=1048576$ shots or $10.7 \mathrm{~s}$ at 40 to $80 \mathrm{~dB}$ SPL in $10 \mathrm{~dB}$ steps. The frequencies 109 were chosen such that each component contained an integer number of points per cycle and the stimulus contained no harmonics ${ }_{110}$ or distortion products up to third order (24). Each sinusoidal component in the complex was assigned a random phase so the 111 pressure magnitude of the complex was $\sim \sqrt{60}$ higher than the magnitude of each component. DPOAEs in response to swept 112 two-tone $\left(f_{1}\right.$ and $\left.f_{2}\right)$ stimuli were measured before each set of tuning curves throughout the experiment. In these measurements, ${ }_{113} f_{2}$ was varied from 1 to $48 \mathrm{kHz}, f_{1}$ and $f_{2}$ were held at a fixed ratio of $f_{2}=1.2 f_{1}$, and the two primary tones were presented at ${ }_{114} 50$ and $70 \mathrm{~dB}$ SPL for $1 \mathrm{~s}$ and averaged 50 times. The noise level was typically $-3-0 \mathrm{~dB}$ SPL for these recordings.

\section{${ }_{115}$ Optical Coherence Tomography and Spectral Domain Phase Microscopy}

${ }_{116}$ Cochleae were imaged with a ThorLabs Telesto III OCT equipped with an LSM03 5×, 0.055 NA objective lens. After the initial ${ }_{117}$ animal surgery, the gerbils were placed under the OCT and the instrument's video camera and an operating microscope were ${ }_{118}$ used to position the head. Recordings were made in the cochlear base near the $25 \mathrm{kHz}$ location, found by aiming the system ${ }_{119}$ apically through the round window. Initially, continuous two-dimensional scanning was done with the ThorImage program and 120 small adjustments made to the position until the OCC was centered in the field of view. The position was adjusted until the two ${ }_{121}$ gaps in the OC corresponding to the inner and outer tunnels (Fig. 1 B) were visible in the B-and A-scans. For vibrometry, the ${ }_{122}$ OCT was controlled with custom software written in C++ based on the ThorLabs software development kit (SDK). Before and ${ }_{123}$ after each set of tuning curves, the OCT acquired $1 \mathrm{~mm}$ wide B-scans and the two images were compared to confirm that the ${ }_{124}$ sample was stable over the course of the measurement.

${ }_{125}$ For vibrometry, a single A-scan through the BM and OHC region was selected and time-locked A-scan spectra were ${ }_{126}$ recorded as the sound played, and then saved to a hard disk as 16 bit raw files for offline analysis. The time series of spectra ${ }_{127}$ (termed M-scan) were converted to a series of complex numbers representing reflectivity versus depth (see (22) for analytical ${ }_{128}$ details). Pixels of local maxima in the time-averaged A-scan magnitude, corresponding to specific features in the OCT image, 129 were selected for further analysis, for example, a pixel on the round window membrane and several pixels in the BM and ${ }_{130} \mathrm{OHC}$ region. In spectral domain phase microscopy, the displacement-vs-time of each pixel in the A-scan is proportional to ${ }_{131}$ the phase-vs-time of the complex M-scan at that pixel. The noise level in the vibration measurement is determined by the 132 magnitude of the selected A-scan pixel, which depends on the feature and reflectivity. In the best preparations, the noise floor ${ }_{133}$ could be as low as $.02-.05 \mathrm{~nm}$. Noise rises if the A-scan peak is reduced (Fig. $3 \mathrm{~A}$ ), which can happen due to micrometer-scale ${ }_{134}$ shifts in the preparation. Several baseline measurements were made before the furosemide to establish a solid baseline and the 135 most complete data set was used for presentation. The vibration and interleaved DPOAE measurements were stable during these ${ }_{136}$ baseline runs; the DPOAE results in Fig. 10 illustrate that stability.

${ }_{137}$ Once the vibration time waveforms were acquired for the regions of interest, the amplitudes and phases at the sound stimulus 138 frequencies were extracted by Fourier analysis. For each stimulus frequency, the response was deemed significant if the Fourier 


\section{Strimbu et al.}

${ }_{139}$ coefficient was 3.2 times larger than the standard deviation of the noise level, measured from ten neighboring bins in the ${ }_{140}$ spectra. (In the presented plots, when individual data points met that criterion but neighboring points did not, they were usually ${ }_{141}$ removed; this removed distracting isolated points that were very close to the defined noise floor.) The resulting tuning curves ${ }_{142}$ are presented in terms of the gain, defined as displacement per unit pressure. The phases are referenced to the ear canal pressure. ${ }_{143}$ Analysis scripts were custom written in Matlab.

144 At the start of an experiment, we tested the displacements at one or more locations by playing a short zwuis complex 145 containing 10 frequencies from 10 to $40 \mathrm{kHz}$ at 60,70 , and $80 \mathrm{~dB}$ for $1 \mathrm{~s}$. We could perform a complete analysis on these short ${ }_{146}$ recordings in approximately five mins and used the resulting coarse tuning curves to gauge the quality of the displacements at 147 the selected location and to estimate the best frequency.

\section{${ }_{148}$ Experimental Paradigm}

149 After the positioning described above we took a series of baseline measurements each consisting of a set of DPOAE audiograms 150 and vibration tuning curves. After these were completed, the gerbil was given an iv injection of furosemide (100 mg/kg) in the 151 left femoral vein. A set of distortion product measurements was taken immediately after the injection. When the injection was 152 successful, the $2 f_{1}-f_{2}$ DPOAEs at $50 \mathrm{~dB}$ typically fell to the noise level and the DPOAEs at $70 \mathrm{~dB}$ were greatly reduced in ${ }_{153}$ amplitude. A set of accompanying zwuis tuning curves was then taken. The measurements were repeated approximately every ${ }_{154} 10 \mathrm{mins}$ for up to four hours post injection. The DPOAE measurements took approximately three mins each and a complete 155 set of tuning curves took approximately four mins, primarily due to the time required, $\sim 40$ s, to transfer large raw files to a 156 solid state drive. Due to the long time required for each experiment, some drift in the preparation was inevitable. Between ${ }_{157}$ recordings, we monitored the position of the cochlea with ThorImage and made minor adjustments to the position as needed. At 158 the conclusion of the experiment, the animals were overdosed with pentobarbital. In some preparations, a set of post mortem 159 tuning curves was acquired after the animal expired.

\section{${ }_{160}$ Electrophysiological Recordings}

${ }_{161}$ The EP and microphonic potentials (termed local cochlear microphonic, LCM) were measured as described previously (15), 162 and the results shown here in Fig. 2 are redrawn from that study, as they provide important background information. The LCM ${ }_{163}$ was measured with an insulated tungsten electrode (tip diameter of $\sim 1 \mu \mathrm{m}$ ) inserted into scala tympani and advanced close to 164 the BM at the base of the cochlea, close to the $18 \mathrm{kHz}$ location. The LCM was measured in response to pure-tone stimuli. The 165 voltages were amplified $500-1000 \times($ PARC EG\&G) and recorded with the TDT system. The EP was measured with an $\sim 10$ ${ }_{166} \mu \mathrm{m}$ diameter glass micro-electrode with an $\mathrm{Ag} / \mathrm{AgCl}$ pellet and filled with $0.5 \mathrm{M} \mathrm{KCl}$. The reference electrode was filled with ${ }_{167}$ standard saline and was placed on the muscle of the right leg. The DC signal was amplified 10× and recorded every second 168 with a usb DAQ board (DATAQ Instruments Inc.).

\section{${ }_{169}$ RESULTS}

170 A total of 13 gerbils, 9 male and 4 female, were used in this study. At the start of the measurements, all exhibited large DPOAEs 171 and baseline OCC vibrations were typical of healthy ears. Five of these 13 animals survived at least two hours post iv-injection of 172 furosemide and showed significant, and in three cases nearly complete, recovery of cochlear amplification at the best frequency. ${ }_{173}$ Of these five experiments, results from the two that showed the most complete recovery are shown in detail and results from 174 two others is shown in grouped data. The third animal that showed nearly complete recovery suffered from mechanical drift in 175 the apparatus, and thus recovery was not successfully tracked. Four animals showed only partial recovery, and died between 50 176 and 100 mins after the injection; results from these is shown individually and grouped. In one experiment, the gerbil survived 177 for several hours post-injection and the vibrations showed a decreased amplitude following furosemide and robust recovery 178 in the sub-BF region but an irreversible loss of amplification at frequencies close to BF. The observed incomplete recovery 179 in several preparations is not unexpected given the invasive nature of the surgery and the long time scale of the experiment. 180 Finally, in three experiments, the iv injection was not successful.

181 The primary measurement of this study was that of vibration within the OCC during recovery from furosemide-induced 182 reduction of EP. Sound stimuli consisted of multi-tone "zwuis" stimuli, in which at each amplitude level, all tones are delivered 183 simultaneously. The tuning observed with multi-tone stimuli is similar to that with pure-tone stimuli, but nonlinearity is more ${ }_{184}$ pronounced, likely due to the increased sound volume $(20,24)$. We concentrated on two regions within the OCC, the BM and 185 the $\mathrm{OHC}$ region, as shown in introductory Fig. 3. The two regions can be found based on the surrounding fluid-filled regions ${ }_{186}$ of the inner and outer tunnels of Corti, which are dark in the B-scan (see Fig. 1B for anatomical labels). The outer tunnel is 187 distinct and the inner tunnel between the pillars is less distinct in this particular B-scan, but still detectable. The vertical line in 188 the B-scan indicates the location of the A-scan where motion was measured, and the SD-OCT technique makes simultaneous 
189 motion measurements at all locations in the A-scan. In Fig. 3E-J motion responses are shown from three points within the BM ${ }_{190}$ region; in Fig. 3K-P responses are shown from three points within the OHC region. The spacing between BM and OHC-region 191 locations was typically $\sim 60 \mu \mathrm{m}$. Both BM and OHC regions are tuned and nonlinear, but the OHC-region responses are up to 192 an order of magnitude larger than BM responses, and compressive nonlinearity in the BM responses begins $\sim 1 / 2$ octave below 193 the BF, whereas in the OHC-region nonlinearity extends throughout the entire frequency range. These findings are similar to ${ }_{194}$ previous measurements obtained by OCT and interferometry $(20,23,25-27)$. The intra-region differences in vibration are 195 small compared to inter-region differences. This is an important point because motion results are most reliable when taken from 196 a local maxima in the A-scan (28) and in the hours-long furosemide study the preparation and image underwent small shifts and 197 therefore the exact same points were not probed during the course of the experiment. Post-mortem responses are shown in Fig. 198 3Q-T; both OHC and BM regions became linear and were either tuned broadly (BM) or nearly low-pass (OHC region).

199 We used furosemide to reversibly reduce the EP as in Fig. 2A and measured OCC vibrations in vivo before and after the 200 introduction of the drug. Recordings were taken immediately after the injection and repeated every ten mins for up to four hours. ${ }_{201}$ Interwoven with the vibration measurements, DPOAEs elicited with equal-level primaries of 50 and $70 \mathrm{~dB}$ SPL were measured. ${ }_{202}$ Data from the two preparations with the fullest recovery are presented in Figs. 4 and 5. These show DPOAE-grams and BM and ${ }_{203}$ OHC-region tuning curves measured at select time points.

${ }_{204}$ Prior to the injection, baseline BM tuning curves and phases were typical of healthy ears (blue curves in $\mathrm{C}$ and $\mathrm{D}$ of ${ }_{205}$ Figs. 4 and 5). The gains showed the characteristic peak at the BF ( $\sim 26 \mathrm{kHz}$ for both of these preparations) a compressive ${ }_{206}$ nonlinearity at nearby frequencies, and linear growth for sub-BF frequencies, starting about 1/2 octave below BF. As in Fig. 3, ${ }_{207}$ OHC-region vibrations (red curves) exhibited higher amplitudes, or equivalently higher gains, than the BM vibrations and ${ }_{208}$ were compressively nonlinear across the entire frequency range tested. The phases showed traveling wave phase accumulation ${ }_{209}$ through more than 4 cycles, with only subtle SPL-dependence. The OHC-region phases led the BM-region phases by .02 210 cycles at $5 \mathrm{kHz}$, reducing to $\sim .01$ cycles at $10 \mathrm{kHz}$ and could lag slightly at frequencies above $20 \mathrm{kHz}$.

${ }_{211}$ Following the furosemide injection, the BM responses resembled those of a passive cochlea (blue curves in panel E of ${ }_{212}$ Figs. 4 and 5). In the BF region there was a loss of amplitude particularly for low and moderate SPL responses, and the gain ${ }_{213}$ curves showed broad, nearly linear tuning. The responses at 40 and $50 \mathrm{~dB}$ SPL often fell below the noise level. The responses in ${ }_{214}$ the linear region below the BF were essentially unaffected by the drug. These observations are similar to previous studies of ${ }_{215}$ the effects of furosemide on BM vibrations (12). Following iv-furosemide, the OHC-region vibrations showed a decrease in ${ }_{216}$ amplitude and the tuning became almost low-pass but remained compressively nonlinear at all frequencies where responses ${ }_{217}$ were detectable. The response phases changed mildly. Amplitude recovery proceeded over several hours. 50 mins post-injection ${ }_{218}$ the BF-region BM and OHC vibrations remained depressed (Figs. 4 and 5I), while sub-BF OHC vibrations had fully recovered ${ }_{219}$ in expt. 785 (Fig. 4I), and substantially recovered in expt. 788 (Fig. 5I). 70 mins after the furosemide treatment, the BF-peak ${ }_{220}$ began to recover - the BF-peak recovery signifies the recovery of cochlear amplification (Figs. 4 and 5K). In expt. 785, both the ${ }_{221} \mathrm{BM}$ and $\mathrm{OHC}$ vibrations had almost fully recovered at $120 \mathrm{mins}$ (Fig. 4M), and in expt. 788, recovery was substantial at 120 222 mins (Fig. 5M). Beyond this time point the responses did not improve.

${ }_{223}$ Prior to the furosemide injection, all the ears tested exhibited robust DPOAEs in response to swept two-tone stimuli. The ${ }_{224} 2 f_{1}-f_{2}$ levels were typically $30-40 \mathrm{~dB}$ lower than the primaries and present up to $32 \mathrm{kHz}$, the maximum frequency tested, 225 when elicited with the $70 \mathrm{~dB}$ SPL primaries. With $50 \mathrm{~dB}$ SPL primaries they were measurably present to $\sim 25 \mathrm{kHz}$. Just after 226 furosemide, DPOAEs dropped into the noise level ( $\sim 0 \mathrm{~dB}$ SPL) at all frequencies for the $50 \mathrm{~dB}$ primaries and at frequencies ${ }_{227}$ above $\sim 15 \mathrm{kHz}$ for the $70 \mathrm{~dB}$ primaries. Following an early burst of recovery at relatively low frequencies (below $10 \mathrm{kHz}$ ), ${ }_{228}$ DPOAEs gradually increased, showed their fullest recovery at $\sim 120 \mathrm{mins}$, similar to the vibration. At that point DPOAEs had 229 recovered fully at frequencies below $\sim 22 \mathrm{kHz}$, which corresponds to $f_{2}$ of $\sim 32 \mathrm{kHz}$. The DPOAE recovery was not monotonic 230 and the time course of the recovery supports the notion that operating point is a key component in the recovery of cochlear ${ }_{231}$ amplification, as will be discussed later.

232 Fig. 6 shows the time variation of the responses at $\mathrm{BF}$ and $\mathrm{BF} / 2$ for the two illustrative experiments. At $\mathrm{BF} / 2$, $\mathrm{BM}$ responses ${ }_{233}$ were unaffected by the furosemide treatment (Fig. $6 \mathrm{C}$ and $\mathrm{G}$ ). In contrast, the $\mathrm{OHC}$ region $\mathrm{BF} / 2$ responses retained nonlinearity ${ }_{234}$ even immediately post-furosemide; the responses dropped slightly (at most a factor of $\sim 4$ ) and gain losses were largest at ${ }_{235}$ high SPL. The OHC-region BF/2 responses recovered nearly fully by 50 mins for expt. 785 (Fig. 6D); for expt. 788 the main ${ }_{236}$ recovery was by $\sim 70$ mins and was not full at high SPL (Fig. 6H). At BF, BM responses dropped most at low SPL and for both ${ }_{237}$ experiments at 40 and $50 \mathrm{~dB}$ SPL they dropped beneath the noise floor (Fig. 6A and E). There was considerable recovery at ${ }_{238} 60-70 \mathrm{mins}$, when these low-SPL responses re-emerged, and continued recovery to 100 mins. The OHC-region BF responses ${ }_{239}$ showed an even greater disappearance into the noise (Fig. 6B and F), and by considering Figs. 4 and 5, this can be seen to be ${ }_{240}$ due to the low-pass characteristic of the post-furosemide OHC-region responses: responses cut off at frequencies below the ${ }_{241} \mathrm{BF}$. In the OHC-region, recovery of amplification at the BF was well underway at $\sim 60-70$ mins, as in the BM region, and ${ }_{242}$ continuing to $100-120$ mins. The time course of amplification recovery as measured in these vibration responses is similar to ${ }_{243}$ what was observed in LCM BF responses (15). To illustrate that similarity, LCM BF data from Fig. 2B are plotted along with 
${ }_{244}$ OHC-region curves in panels B and F. (The LCM data were scaled to overlie the vibration data, which does not affect the 245 comparison since the $y$-axis is logarithmic.)

246 In four preparations, recovery following furosemide never became substantial. Two examples are in Fig. 7. The reductions ${ }_{247}$ just post furosemide in these two preparations are similar to those in Figs. 4 and 5. In particular, the sub-BF nonlinearity is ${ }_{248}$ retained in these preparations just after furosemide, while amplification is absent. However, after faltering steps to recovery, ${ }_{249}$ amplification declined in these preparations. Fig. 8 contrasts the recovery of BF/2 responses averaged over the four animals with ${ }_{250}$ good recovery, and four animals with poor recovery. The initial drop in responses is similar, as is the initial recovery at 20 ${ }_{251}$ mins, but at 50 mins the "good" preparations are improving, while in the poor preparations recovery has turned into decline. ${ }_{252}$ The incomplete recovery in some preparations is likely due to a general decline in cochlear condition, which often happens in ${ }_{253}$ experimental cochlear physiology.

${ }_{254}$ Returning to the preparations with good recovery, we consider the changes and recovery in the phase responses. Data are ${ }_{255}$ shown from the two experiments in Figs. 4 and 5 and a third experiment (783). The top row of Fig. 9 (A, C, E) shows baseline ${ }_{256}$ phase data and illustrates that BM and OHC-region phases go through a similar traveling wave excursion of several cycles. The ${ }_{257}$ second row (B, D, F) explores the phase differences, and how they change in time. In the baseline condition at low frequency (a ${ }_{258}$ few $\mathrm{kHz}$ ) OHC-region vibration phase leads BM phase by up to $\sim 0.2$ cycle. This lead declines with frequency to eventually 259 become a lag of $\sim 0.1$ cycle close to the BF. Just after furosemide, the OHC-BM phase difference dropped by $\sim .05$ cycle $\left(18^{\circ}\right)$ ${ }_{260}$ across frequency in the data of Fig. 9 B, and that observation approximately holds for the other two preparations in D and F. ${ }_{261}$ During the recovery period, that general-frequency drop in OHC-BM phase difference was partially but never fully reversed. In ${ }_{262}$ Fig. $9 \mathrm{G}$ phase differences within each region before and just after furosemide are shown. This is grouped data, with mean ${ }_{263}$ and standard deviation from all eight preparations. The BM phase changed little and thus most of the time-dependent phase ${ }_{264}$ differences seen in Fig. 9 B, D and F resulted from changes in the OHC-region phase.

\section{${ }_{265}$ DISCUSSION}

${ }_{266}$ The electrical gradient of the EP provides much of the driving force for the MET currents. In the OHCs, the receptor potentials ${ }_{267}$ generated by these currents drive electromotility which boosts BM and reticular lamina vibrations and is an essential component ${ }_{268}$ of the cochlea's active process. We used furosemide to reduce EP and followed the recovery of OCC vibrations and DPOAEs 269 over several hours.

270 $\mathrm{BM}$ and $\mathrm{OHC}$-region vibrations recovered nearly fully in several preparations, and the time-scale of recovery in different ${ }_{271}$ frequency regions and in DPOAEs indicates that several processes are involved in recovery. OHC-region sub-BF vibration 272 retained nonlinearity throughout, and was back to baseline by $\sim 50-70$ mins, signifying relatively rapid recovery of robust ${ }_{273}$ electromotility. The recovery of the BF peak in OHC-regions and at the BM, signifying functional cochlear amplification, ${ }_{274}$ occurred later, mainly during the time period between 70 and 120 mins. This two-stage recovery is reminiscent of the findings 275 of Wang et al., who studied changes in LCM following the same iv-furosemide protocol (15). In that study, EP had stabilized at ${ }_{276}$ a sub-normal level at $\sim 40$ mins and the LCM BF peak finally recovered at 100 mins or later. The timing of changes in LCM ${ }_{277}$ second harmonic responses indicated that the recovery of cochlear amplification occurred simultaneously with re-centering of ${ }_{278}$ the MET channel operating point. In the present study of OCC vibration, the comparable noise floor was higher than that of the ${ }_{279}$ LCM study, and second harmonics could not be examined. DPOAEs were used to explore operating point shifts in the present 280 study.

${ }_{281}$ The recovery of DPOAEs in Figs. 4 and 5 is nonmonotonic, and a boost of recovery at $\sim 70$ mins is apparent. To observe this ${ }_{282}$ more fully, in Fig. 10 the recovery time course of an average of three DPOAEs at adjacent frequencies close to the BF of the ${ }_{283}$ measurement location are shown as line plots. Fig. $10 \mathrm{~A}$ and B are from the preparations in Figs. 4 and 5; Fig. $10 \mathrm{C}$ is a mean ${ }_{284}$ of the four preparations in which amplification recovered most fully. The reduction at $t=0$ corresponds to the dark line that ${ }_{285}$ appears at $t=0$ in the heat plots of Figs. 4 and 5. Following that sharp reduction, the DPOAEs underwent 20 mins of recovery, ${ }_{286}$ but then dropped again, reached a local minimum at 50 mins and then entered a final recovery phase. The size of DPOAEs is ${ }_{287}$ expected to be directly affected by the reduction in EP, which would reduce OHC receptor current and voltage, and thus reduce ${ }_{288}$ active cochlear feedback. However, the nonmonotonic recovery illustrated in Fig. 10 cannot be attributed to the EP, which in our 289 experience recovers monotonically and had likely stabilized at $\sim 40$ mins post-furosemide (15).

${ }_{290}$ These results can be considered through the lens of the MET operating point behavior found in (15). In Fig. $11 \mathrm{~A}$, the ${ }_{291}$ MET function that was derived in (15) is shown with zero operating point offset, and with offsets of -0.12 and $+.05 \mathrm{~Pa}$. These 292 offsets are included because in the findings shown in Fig. $2 \mathrm{C}$, the MET operating point was positive at a value of $\sim .05 \mathrm{~Pa}$ at $293 \mathrm{t}=0$, shifted negative to a minimum value of $\sim-0.12 \mathrm{~Pa}$ at $\sim 60 \mathrm{mins}$, and began to recenter (shift towards zero) at $\sim 60-70$ 294 mins. Fig. 11 B shows how the size of the $2 f_{1}-f_{2}$ distortion produced by the nonlinearity in Fig. $11 \mathrm{~A}$ is affected by operating ${ }_{295}$ point shifts $(7,29)$. The primary inputs into the nonlinearity were at a level of $70 \mathrm{~dB}$ SPL (.06 Pa peak), to coincide with the ${ }_{296}$ primary inputs producing the DPOAEs in Fig. 10. Fig. 11 B shows that the $2 f_{1}-f_{2}$ distortion product will be a maximum with 
${ }_{297} 0$ offset, will reduce to zero at an offset of $\sim \pm .012 \mathrm{~Pa}$, and increase for larger offsets. Thus, the second minimum in the DPOAE ${ }_{298}$ responses, starting its descent at $\sim 25$ mins in Fig. 10, and second boost of DPOAE recovery starting at $\sim 50$ mins, is likely due 299 to MET operating point shift. The appearance of a local minimum in the DPOAE at 50 mins is predicted for an operating point 300 that had shifted to its most negative value at $50 \mathrm{mins}$, and shifted back toward zero between 50 and 100 mins. It is confirming ${ }_{301}$ that this is the MET behavior that was observed in (15), redrawn in this paper's Fig. 2 C. It is also worth underlining that 302 between 50 and 100 mins is also when the BF peak - signifying cochlear amplification - underwent its steady recovery in BM ${ }_{303}$ and OHC-region responses.

${ }_{304}$ This combination of findings focuses us on the questions: What is required for cochlear amplification? What is essential, 305 and what is sufficient? We showed in (15) that a normal EP is not essential, and recovery of cochlear amplification at sub-normal ${ }_{306}$ EP had been hypothesized previously based on DPOAE recovery (16). OHC electromotility is essential based on genetic studies ${ }_{307}$ that changed prestin to a non-motile form (30). However, from the present study, the mere presence of robust electromotility is 308 not sufficient for amplification of the BF-peak.

${ }_{309}$ Simultaneous pressure and voltage measurements found that a phase shift between LCM and BM displacement was critical 310 for OHC forces to be phased to provide amplification (19), and we explored the present data for time-dependence in the vibration ${ }_{311}$ phase following furosemide and recovery (Fig. 9 B, D, F.) As a broad-brush observation from these experiments, following ${ }_{312}$ furosemide the OHC-BM phase difference was offset by $\sim-.05$ cycle $\left(-18^{\circ}\right)$. This offset reduced the sub-BF phase lead of ${ }_{313}$ OHC-region relative to BM and increased the BF-region phase lag of OHC-region relative to BM. During the recovery of 314 amplification, the most compelling phase recovery came from expt. 788 (Fig. 9 B) in which, in the 70-120 mins during which 315 amplification recovered, this $18^{\circ}$ furosemide-induced offset fully reversed at frequencies above $13 \mathrm{kHz}$. Thus, in this experiment 316 the phasing between $\mathrm{OHC}$ and $\mathrm{BM}$ motion returned to a pre-furosemide character, concurrent with recovery of amplification. ${ }_{317}$ Expt. 785 also underwent a furosemide-induced negative offset in OHC-BM phase, and the offset recovered but only partially, ${ }_{318}$ although amplification showed substantial recovery. Expt. 783 also underwent a furosemide-induced negative offset in OHC-BM ${ }_{319}$ phase and in this case the offset recovered barely at all. In expt. 783, amplification recovery, while substantial, was less full ${ }_{320}$ than in the other two preparations. In sum, an OHC-BM phase change was robust following furosemide - a downward offset ${ }_{321}$ occurred between the baseline and $t=0$ measurements in three of three explored data sets. Recovery of that offset was only 322 weakly correlated with recovery of amplification. In the study of Cooper et al. (23) the phase of OHC vibration relative to BM ${ }_{323}$ was found to be viewing-angle dependent, and subtle preparation-viewing-angle differences could conceivably be affecting the 324 repeatablity of phase changes in our study.

325 Based on the results in this study and its predecessor (15), the recovery of amplification occurs well after full recovery of ${ }_{326}$ electromotility and stabilization of EP, and concurrent with MET operating point recentering. These findings lend strength to the ${ }_{327}$ expectation that beyond the size of critical factors such as driving voltage and electromotility, amplification relies on qualitative ${ }_{328}$ conformational factors. That concept was illuminated by Jacob and Fridberger (31), who applied quasi-static current injections 329 to restore the EP in situ in guinea pig temporal bone preparations. Using confocal fluorescent microscopy and interferometry ${ }_{330}$ they found that the organ of Corti underwent a series of mechanical conformational changes, shifting the apical region of the ${ }_{331}$ OHCs towards scala vestibuli, and these shifts positioned the system into a region of increased sensitivity. The position changes, ${ }_{332} \sim 50-100 \mathrm{~nm}$, are smaller than the resolution of an OCT when used in two-dimensional imaging mode, so we cannot probe ${ }_{3 з 3}$ those findings directly with our imaging system. The recovery of amplification we observed occurred after EP was expected to 334 be substantially recovered and stabilized, whereas the changes observed by Jacob et al. were concurrent with directly-induced ${ }_{335}$ EP changes. Despite this difference, the finding of that study, that conformational changes play a large role in setting the proper 336 mechanical conditions for amplification, is supported by our findings.

${ }_{337}$ Disrupting the EP could cause other perturbations that lead to operational changes that diminish auditory sensitivity, ${ }_{338}$ and these perturbations could recover on a different time scale than EP. In a recent review article, Manley (32) discusses зз9 the possibility that the EP exists at least in part to drive calcium metabolism in the mammalian cochlea, where evolutionary ${ }_{340}$ pressures caused the endolymphatic calcium concentration to fall to the micromolar level, a value significantly lower than ${ }_{341}$ in the auditory organs of other vertebrates. Calcium plays a number of roles in the hair cells including regulating the open ${ }_{342}$ probability of the MET channels, and may control one or more of the adaptation processes that partially set the dynamic ${ }_{343}$ range of the transduction process (33-35). Reduced EP will drive $\mathrm{Ca}^{2+}$ into scala media, which could affect the TM and its ${ }_{344}$ relationship to hair cells (36). In an in situ study, Strimbu et al. found that the calcium concentration in the TM is elevated ${ }_{345}$ relative to the bulk endolymph by $\sim 25 \mu \mathrm{M}$ (37). In that study, following a brief exposure to loud sounds, both the TM calcium ${ }_{346}$ and cochlear microphonic were reduced and recovered in synchrony on a time-scale of $\sim 30$ mins. Using organ of Corti explants, ${ }_{347}$ Vélez-Ortega et al. showed that reducing the MET currents with channel blockers and manipulation of both intracellular and ${ }_{348}$ extracellular $\mathrm{Ca}^{2+}$ for as little as one hour could alter the morphology of the hair bundles. Reducing calcium entry into the ${ }_{349}$ bundles caused contraction of the shorter rows that contain the transduction channel and its associated machinery (38). If a 350 similar effect occurs in vivo in an adult animal with fully mature hair cells, deleterious changes could occur to the hair bundles ${ }_{351}$ during the $30-40$ mins following the furosemide injection when the EP remains significantly depressed. One would expect 
Strimbu et al.

352 such physical changes to recover over a longer time scale than the recovery of EP.

${ }_{353}$ In addition to reducing the $\mathrm{EP}$, furosemide may reduce auditory sensitivity through other mechanisms. Loop diuretics 354 weaken the blood labyrinth barrier by damaging the tight junctions lining the stria vascularis, an effect that has been exploited to 355 enhance the uptake of contrast agents in MRI studies (39). The ototoxicity of other agents, such as cisplatin or aminoglycosides, 356 is known to increase when administered simultaneously with loop diruetics, such as furosemide, an effect that presumably ${ }_{357}$ arises due to the increased permeability of the drugs in the perilymphatic spaces of the cochlea (40). Furosemide applied ${ }_{358}$ extracellularly to isolated outer hair cells diminishes nonlinear capacitance, a surrogate for electromotility (41). More generally, 359 furosemide is known to affect the biophysical properties of lipids in vitro (42). Such changes to lipid bilayer of the hair cells' ${ }_{360}$ bodies could alter electromotilty or the properties of the MET channels themselves $(43,44)$. However, the concentrations of ${ }_{361}$ furosemide in perilymph (45) and endolymph (46) following iv injections at the dosages used in this study have been measured ${ }_{362}$ and are in the micromolar range, approximately an order of magnitude lower than the extracellular concentration needed to ${ }_{363}$ evoke changes in nonlinear capacitance (41) or lipid properties in vitro (42). This suggests that any direct affects furosemide ${ }_{364}$ might have on the $\mathrm{OHC}$ bodies would be secondary in importance to the reduction of the EP.

${ }_{365}$ In summary, we used the reversible changes caused by iv-furosemide to probe the essential ingredients of cochlear ${ }_{366}$ amplification. Following furosemide treatment the BF peak disappeared in BM and OHC-region mechanical responses while ${ }_{367}$ the sub-BF OHC-region nonlinearity was retained. Sub-BF OHC-region responses substantially or fully recovered to baseline ${ }_{368}$ levels during the first hour following furosemide. This suggests that $\mathrm{OHC}$ electromotility was fully operational at that point, ${ }_{369}$ and based on recent findings from our group, EP would have been stable at a sub-normal level. The BF peak underwent its 370 own significant recovery during the second hour following furosemide, concurrent with an apparent re-centering of MET ${ }_{371}$ channels (15). These findings indicate that the presence of normal, high EP is not necessary for amplification and robust OHC 372 electromotility is not by itself sufficient for cochlear amplification, and support the idea that the mechanical conformation of the 37з organ of Corti complex is key to a functioning cochlear amplifier.

\section{${ }_{374}$ AUTHOR CONTRIBUTIONS}

${ }_{375}$ C.E.S. Performed OCT experiments, analyzed the data and drafted the manuscript. Y.W. Performed iv injections in initial ${ }_{376}$ experiments, and contributed to study design. E.O. Supervised research, analyzed data and participated in manuscript writing.

\section{${ }_{377}$ ACKNOWLEDGMENTS}

${ }_{378}$ This work was funded by NIH Grant R01-DC015362 and the Emil Capita Foundation.

\section{REFERENCES}

1. Ashmore, J., 2008. Cochlear outer hair cell motility. Physiol. Rev. 88:173-210.

2. Robles, L., and M. A. Ruggero, 2001. Mechanics of the mammalian cochlea. Physiol. Rev. 81:1305-1352.

3. Hudspeth, A. J., 2008. Making an effort to listen: mechanical amplification in the ear. Neuron 59:530-545.

4. Ashmore, J., P. Avan, W. E. Brownell, P. Dallos, K. Dierkes, R. Fettiplace, K. Grosh, C. M. Hackney, A. J. Hudspeth, F. Julicher, B. Lindner, P. Martin, J. Meaud, C. Petit, J. Santos-Sacchi, J. R. Sacchi, and B. Canlon, 2010. The remarkable cochlear amplifier. Hear. Res. 266:1-17.

5. Peng, A. W., and A. J. Ricci, 2011. Somatic motility and hair bundle mechanics, are both necessary for cochlear amplification? Hear. Res. 273:109-122.

6. Fettiplace, R., and K. X. Kim, 2014. The physiology of mechanoelectrical transduction channels in hearing. Physiol. Rev. 94:951-986.

7. Brown, D. J., J. J. Hartsock, R. M. Gill, H. E. Fitzgerald, and A. N. Salt, 2009. Estimating the operating point of the cochlear transducer using low-frequency biased distortion products. J. Acoust. Soc. Am. 125:2129-2145.

8. Lukashin, A., and I. Russell, 1997. A descriptive model of the receptor potential nonlinearities generated by the hair cell mechanoelectric transducer. J. Acoust. Soc. Am. 103:973-980.

9. Keithley, E. M., 2019. Pathology and mechanisms of cochlear aging. J. Neurosci. Res. 0. 
395 10. Tu, N. C., and R. A. Friedman, 2018. Age-related hearing loss: Unraveling the pieces. Laryngoscope Investig Otolaryngol $396 \quad 3: 68-72$.

397 11. Yamasoba, T., F. R. Lin, S. Someya, A. Kashio, T. Sakamoto, and K. Kondo, 2013. Current concepts in age-related hearing loss: epidemiology and mechanistic pathways. Hear. Res. 303:30-38.

399 12. Ruggero, M. A., and N. C. Rich, 1991. Furosemide alters organ of corti mechanics: evidence for feedback of outer hair $400 \quad$ cells upon the basilar membrane. J. Neurosci. 11:1057-1067.

401 13. Sewell, W. F., 1984. The effects of furosemide on the endocochlear potential and auditory-nerve fiber tuning curves in cats. Hear. Res. 14:305-314.

403 14. Komune, S., and T. Morimitsu, 1985. Dissociation of the cochlear microphonics and endocochlear potential after injection of ethacrynic acid. Arch Otorhinolaryngol 241:149-156.

405 15. Wang, Y., E. Fallah, and E. S. Olson, 2019. Adaptation of Cochlear Amplification to Low Endocochlear Potential. Biophys. J. 116:1769-1786.

407 16. Mills, D. M., S. J. Norton, and E. W. Rubel, 1993. Vulnerability and adaptation of distortion product otoacoustic emissions to endocochlear potential variation. J. Acoust. Soc. Am. 94:2108-2122. https://doi . org/10.1121/1.407483.

${ }_{409}$ 17. Schmiedt, R. A., H. Lang, H. O. Okamura, and B. A. Schulte, 2002. Effects of furosemide applied chronically to the round window: a model of metabolic presbyacusis. J. Neurosci. 22:9643-9650.

${ }_{411}$ 18. Fridberger, A., J. B. de Monvel, J. Zheng, N. Hu, Y.Zou, T. Ren, and A. Nuttall, 2004. Organ of Corti Potentials and the Motion of the Basilar Membrane. J of Neuroscience 24:10057-10063. https: //www . jneurosci . org/content/24/45/10057.

${ }_{413}$ 19. Dong, W., and E. S. Olson, 2013. Detection of cochlear amplification and its activation. Biophys. J. 105:1067-1078.

${ }_{414}$ 20. Fallah, E., C. E. Strimbu, and E. S. Olson, 2019. Nonlinearity and amplification in cochlear responses to single and 415 multi-tone stimuli. Hear. Res. 377:271-281.

416 21. Saremi, A., and S. Stenfelt, 2013. Effect of metabolic presbyacusis on cochlear responses: a simulation approach using a physiologically-based model. J. Acoust. Soc. Am. 134:2833-2851.

${ }_{418} 22$. Lin, N. C., C. E. Strimbu, C. P. Hendon, and E. S. Olson, 2018. Adapting a commercial spectral domain optical coherence tomography system for time-locked displacement and physiological measurements. AIP Conference Proceedings 1965:080004.

${ }_{421}$ 23. Cooper, N. P., A. Vavakou, and M. van der Heijden, 2018. Vibration hotspots reveal longitudinal funneling of sound-evoked ${ }_{422}$ motion in the mammalian cochlea. Nat Commun 9:3054.

${ }_{423} 24$. Versteegh, C. P., and M. van der Heijden, 2012. Basilar membrane responses to tones and tone complexes: nonlinear effects ${ }_{424}$ of stimulus intensity. J. Assoc. Res. Otolaryngol. 13:785-798.

${ }_{425} 25$. Chen, F., D. Zha, A. Fridberger, J. Zheng, N. Choudhury, S. L. Jacques, R. K. Wang, X. Shi, and A. L. Nuttall, 2011. A differentially amplified motion in the ear for near-threshold sound detection. Nat. Neurosci. 14:770-774.

${ }_{427} 26$. Lee, H. Y., P. D. Raphael, A. Xia, J. Kim, N. Grillet, B. E. Applegate, A. K. Ellerbee Bowden, and J. S. Oghalai, 2016. Two-Dimensional Cochlear Micromechanics Measured In Vivo Demonstrate Radial Tuning within the Mouse Organ of Corti. J. Neurosci. 36:8160-8173.

430 27. Ren, T., W. He, and D. Kemp, 2016. Reticular lamina and basilar membrane vibrations in living mouse cochleae. Proc. ${ }_{431} \quad$ Natl. Acad. Sci. U.S.A. 113:9910-9915.

432 28. Lin, N. C., C. P. Hendon, and E. S. Olson, 2017. Signal competition in optical coherence tomography and its relevance for ${ }_{433}$ cochlear vibrometry. J. Acoust. Soc. Am. 141:395-405. https://doi.org/10.1121/1.4973867.

434 29. Sirjani, D. B., A. N. Salt, R. M. Gill, and S. A. Hale, 2004. The influence of transducer operating point on distortion 435 generation in the cochlea. J. Acoust. Soc. Am. 115:1219-1229. 
Strimbu et al.

436 30. Dallos, P., X. Wu, M. A. Cheatham, J. Gao, J. Zheng, C. T. Anderson, S. Jia, X. Wang, W. H. Cheng, S. Sengupta, D. Z. ${ }_{437} \mathrm{He}$, and J. Zuo, 2008. Prestin-based outer hair cell motility is necessary for mammalian cochlear amplification. Neuron 438 $\quad 58: 333-339$.

439 31. Jacob, S., M. Pienkowski, and A. Fridberger, 2011. The endocochlear potential alters cochlear micromechanics. Biophys. J. $440 \quad 100: 2586-2594$.

441 32. Manley, G. A., 2017. The mammalian Cretaceous cochlear revolution. Hear. Res. 352:23 - 29. http://www. $442 \quad$ sciencedirect.com/science/article/pii/S0378595516305275, annual Reviews 2017.

443 33. Peng, A. W., T. Effertz, and A. J. Ricci, 2013. Adaptation of mammalian auditory hair cell mechanotransduction is $444 \quad$ independent of calcium entry. Neuron 80:960-972.

445 34. Corns, L. F., S. L. Johnson, C. J. Kros, and W. Marcotti, 2014. Calcium entry into stereocilia drives adaptation of the ${ }_{446}$ mechanoelectrical transducer current of mammalian cochlear hair cells. Proc. Natl. Acad. Sci. U.S.A. 111:14918-14923.

447 https://www.pnas.org/content/111/41/14918.

448 35. Caprara, G. A., A. A. Mecca, Y. Wang, A. J. Ricci, and A. W. Peng, 2019. Hair Bundle Stimulation Mode Modifies $449 \quad$ Manifestations of Mechanotransduction Adaptation. J. of Neurosci. 39:9098-9106.

450 36. Freeman, D. M., K. Masaki, A. R. McAllister, J. L. Wei, and T. F. Weiss, 2003. Static material properties of the 451 tectorial membrane: a summary. Hear. Res. 180:11 - 27. http://www. sciencedirect.com/science/article/pii/ $452 \quad$ S0378595503000728.

453 37. Strimbu, C. E., S. Prasad, P. Hakizimana, and A. Fridberger, 2019. Control of hearing sensitivity by tectorial membrane 454 calcium. Proc. Natl. Acad. Sci. U.S.A. 116:5756-5764. https://www. pnas.org/content/116/12/5756.

${ }_{455}$ 38. Vélez-Ortega, A. C., M. J. Freeman, A. A. Indzhykulian, J. M. Grossheim, and G. I. Frolenkov, 2017. Mechanotransduction 456 current is essential for stability of the transducing stereocilia in mammalian auditory hair cells. eLife 6:e24661. 457 https://doi.org/10.7554/eLife.24661.

458 39. Videhult Pierre, P., J. E. Rasmussen, S. Nikkhou Aski, P. Damberg, and G. Laurell, 2020. High-Dose Furosemide 459 Enhances the Magnetic Resonance Signal of Systemic Gadolinium in the Mammalian Cochlea. Otol. Neurotol. 41:545-553. 460 https://wwW.overleaf.com/project/566b68aa1d7510001698d90.

461 40. Ding, D., H. Liu, W. Qi, H. Jiang, Y. Li, X. Wu, H. Sun, K. Gross, and R. Salvi, 2016. Ototoxic effects and mechanisms of $462 \quad$ loop diuretics. J. Otol. 11:145-156.

${ }_{463} 41$. Santos-Sacchi, J., M. Wu, and S. Kakehata, 2001. Furosemide alters nonlinear capacitance in isolated outer hair cells. ${ }_{464}$ Hear. Res. 159:69 - 73. http://www.sciencedirect.com/science/article/pii/S0378595501003215.

465 42. Bach, D., C. Vinkler, I. R. Miller, and S. R. Caplan, 1988. Interaction of furosemide with lipid membranes. J. Membr. Biol. $466 \quad 101: 103-111$.

${ }_{467}$ 43. Peng, A. W., R. Gnanasambandam, F. Sachs, and A. J. Ricci, 2016. Adaptation Independent Modulation of Auditory Hair ${ }_{468} \quad$ Cell Mechanotransduction Channel Open Probability Implicates a Role for the Lipid Bilayer. J. Neurosci. 36:2945-2956.

${ }_{469}$ 44. Gianoli, F., T. Risler, and A. S. Kozlov, 2017. Lipid bilayer mediates ion-channel cooperativity in a model of hair-cell $470 \quad$ mechanotransduction. Proc. Natl. Acad. Sci. U.S.A. 114:E11010-E11019. https://wWw. pnas. org/content/114/51/ $471 \quad$ E11010.

472 45. Rybak, L. P., T. P. Green, S. K. Juhn, T. Morizono, and B. L. Mirkin, 1979. Elimination Kinetics of Furosemide in ${ }_{473} \quad$ Perilymph and Serum of the Chinchilla: Neuropharmacologic Correlates. Acta Oto-Laryngologica 88:382-387.

${ }_{474}$ 46. Hara, A., K. Machiki, M. Senarita, M. Komeno, and J. Kusakari, 1993. Pharmacokinetics of furosemide in endolymph. $475 \quad$ Auris Nasus Larynx 20:247-254.

${ }_{476}$ Figures and Tables 


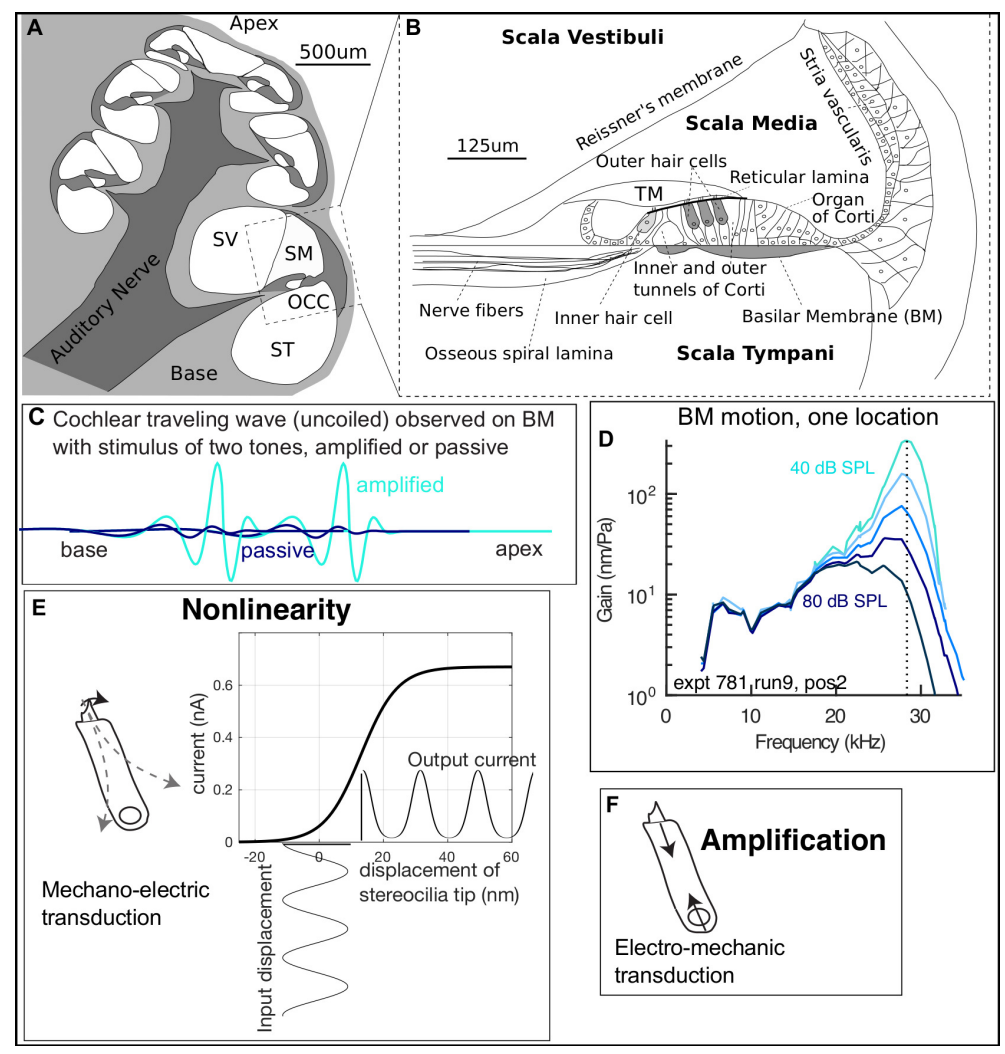

Figure 1: (A) Cross-sectional sketch of the gerbil cochlea, with fluid compartments scala media (SM), scala tympani (ST) and scala vestibuli (SV) labelled. (B) The boxed section in A is expanded, to show the organ of Corti complex (OCC). The OCC is composed of the sensory tissue of the OC (including the inner and outer hair cells (IHC and OHC)) and surrounding acellular structures of the basilar and tectorial membranes (BM and TM). (C) Cartoon of the motion response to two tones on the BM, showing the characteristic amplification in the region where the responses peak, and illustrating that amplification will make otherwise undetectable signals detectable and enhance frequency resolution. (D) BM motion data in a healthy cochlea, showing the hallmarks of amplification: nonlinearity and sharp tuning at low to moderate stimulus levels. The best frequency (BF) is indicated by the vertical dashed line. (E) Nonlinearity is based in the saturation of OHC current when MET channels are pushed to the nearly fully open and fully closed states. OHC current data and Boltzmann function fit redrawn from (6). (F) The mechanical basis of amplification is most likely $\mathrm{OHC}$ somatic forces arising from the prestin molecule's conformation changes following electro-mechanic transduction. 
Strimbu et al.
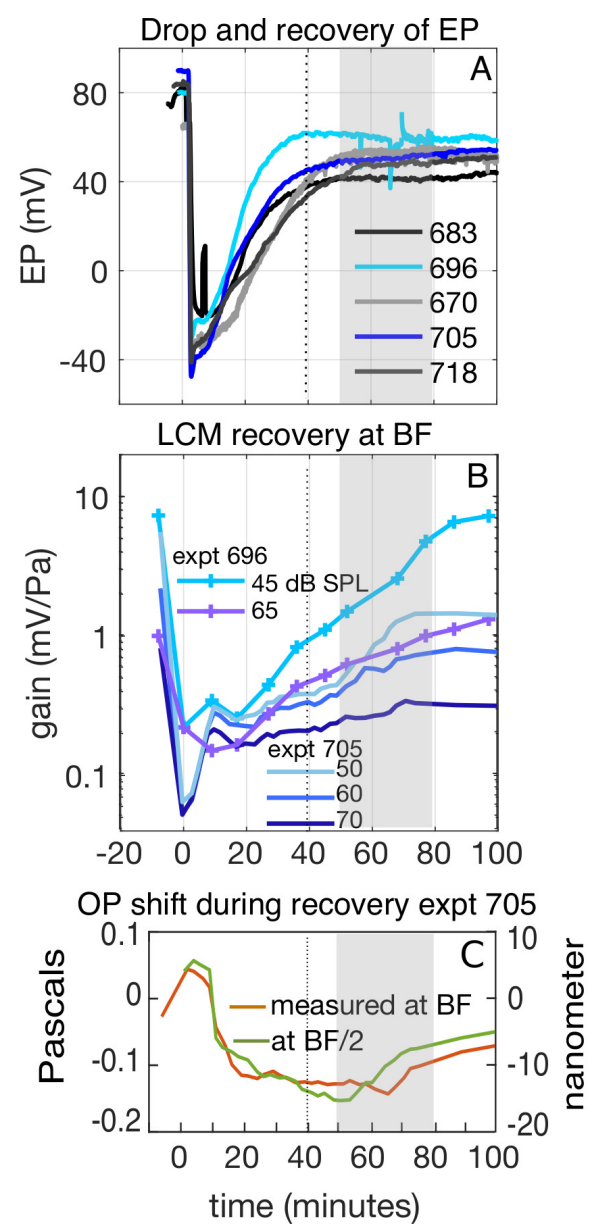

Figure 2: Background results from a recent iv-furosemide study, redrawn from (15). (A) Following iv-furosemide, EP dropped immediately to $-20--40 \mathrm{mV}$, began recovering within $10 \mathrm{mins}$ and stabilized at a sub-normal level $\sim 40$ mins after furosemide delivery (vertical dotted line). The two blue traces correspond to the experiments in (B). (B) Recovery of LCM responses at BF. LCM recovered more slowly than EP, and underwent a substantial recovery in a 50-80 min time frame where EP had fully stabilized but at a sub-normal level (gray rectangles). In expt. 705 LCM responses were measured at relatively dense time points. (C) MET operating point shifts measured in expt. 705, using second harmonic LCM responses. Left axis corresponds to stimulus pressure in the ear canal, right axis is approximate stereocilia bundle shift. Responses at two stimulus frequencies are shown to demonstrate similarity across frequency. The operating point returns towards zero in the 50-80 min time frame coinciding with the recovery of BF LCM in (B). The size and timing of this operating point variation are useful background information for analyzing the vibration and DPOAE results of the present paper. ((15) used single-tone stimuli; LCM data with multi-tone stimuli (but without iv-furosemide) is in (20).) 
bioRxiv preprint doi: https://doi.org/10.1101/2020.05.11.089789; this version posted May 14, 2020. The copyright holder for this preprint (which was not certified by peer review) is the author/funder, who has granted bioRxiv a license to display the preprint in perpetuity. It is made available under aCC-BY-NC-ND 4.0 International license.

Recovery of Cochlear Amplification
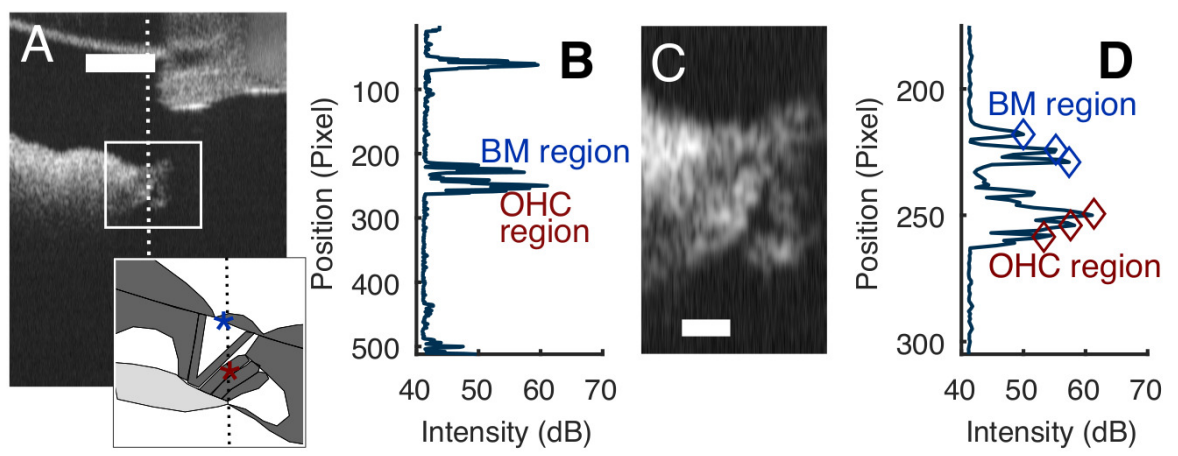

$\mathrm{BM}$ region
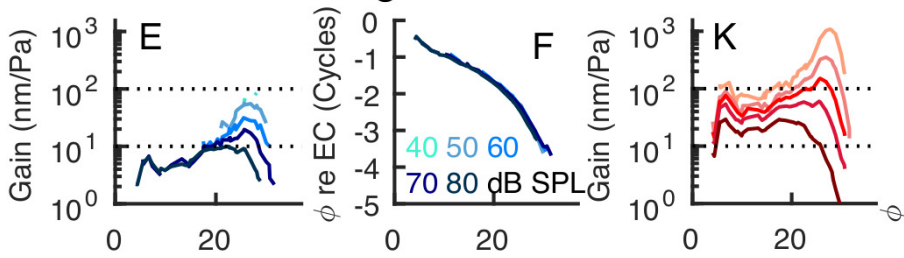

$\mathrm{OHC}$ region
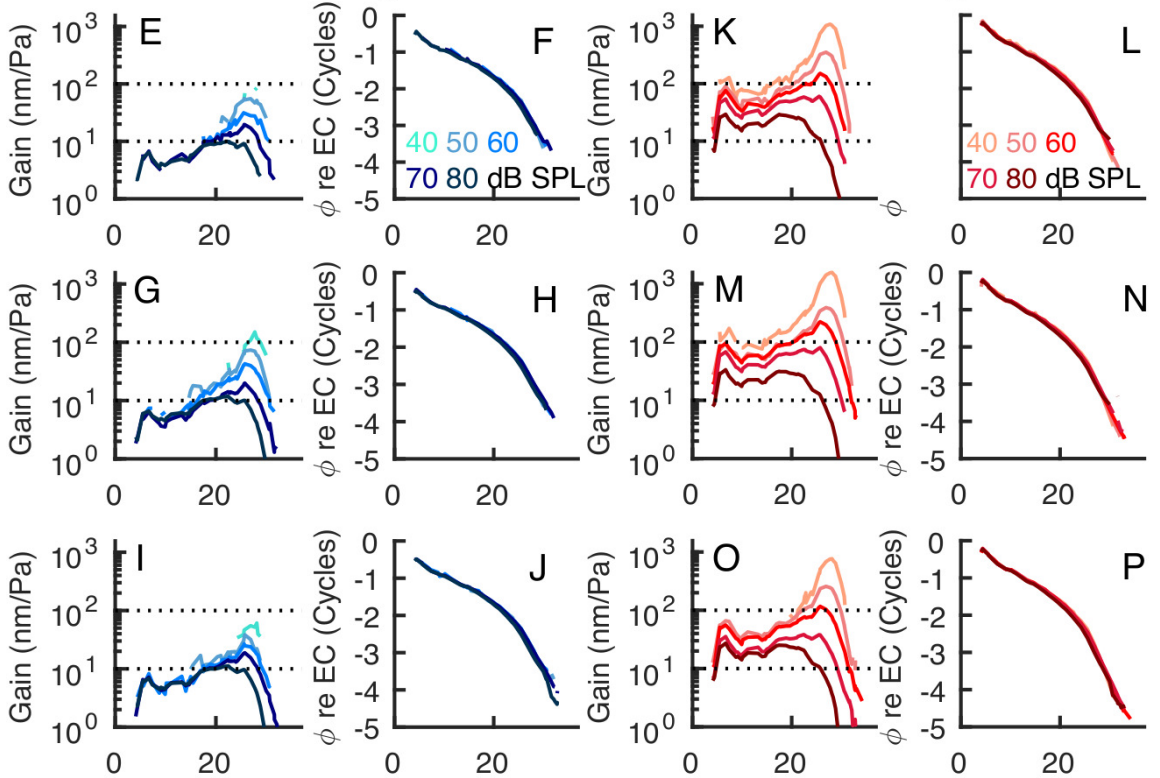

post mortem
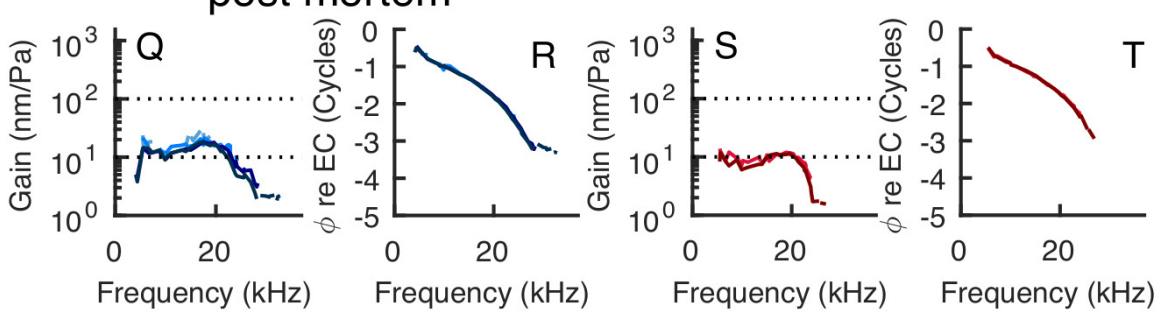

Figure 3: (A) B-scan with sketch showing OHC and BM regions, which were identified using the dark fluid gaps in the B-scan and known anatomy. (Sketch in lower right corner is upside-down and simplified version of Fig.1B. Blue and maroon stars indicate $\mathrm{BM}$ and $\mathrm{OHC}$ regions.) Scale bar $250 \mu \mathrm{m}$. (B) A-scan corresponding to the vertical line in A; this is the line along which vibration measurements were made. The A-scan shows peaks at the round window membrane (top of A-scan) and in the $\mathrm{BM}$ and $\mathrm{OHC}$ regions. The distance between adjacent pixels is $\sim 3 \mu \mathrm{m}$. (C) Expanded view of B-scan. (D) Expanded view of A-scan with locations where vibration was analyzed identified with diamonds. (E-J) Gain and phase re: EC pressure from the three identified points in the BM region. Darker shades indicate higher sound pressure levels, key in panels F and L. K-P Gain and phase: re: EC pressure from the three identified points in the OHC region. (Q-T) Post-mortem responses in the two regions. Only the 70 and $80 \mathrm{~dB}$ stimuli gave displacements that were significantly above the noise threshold. Expt. 789, 10-2-2019. 
Strimbu et al.
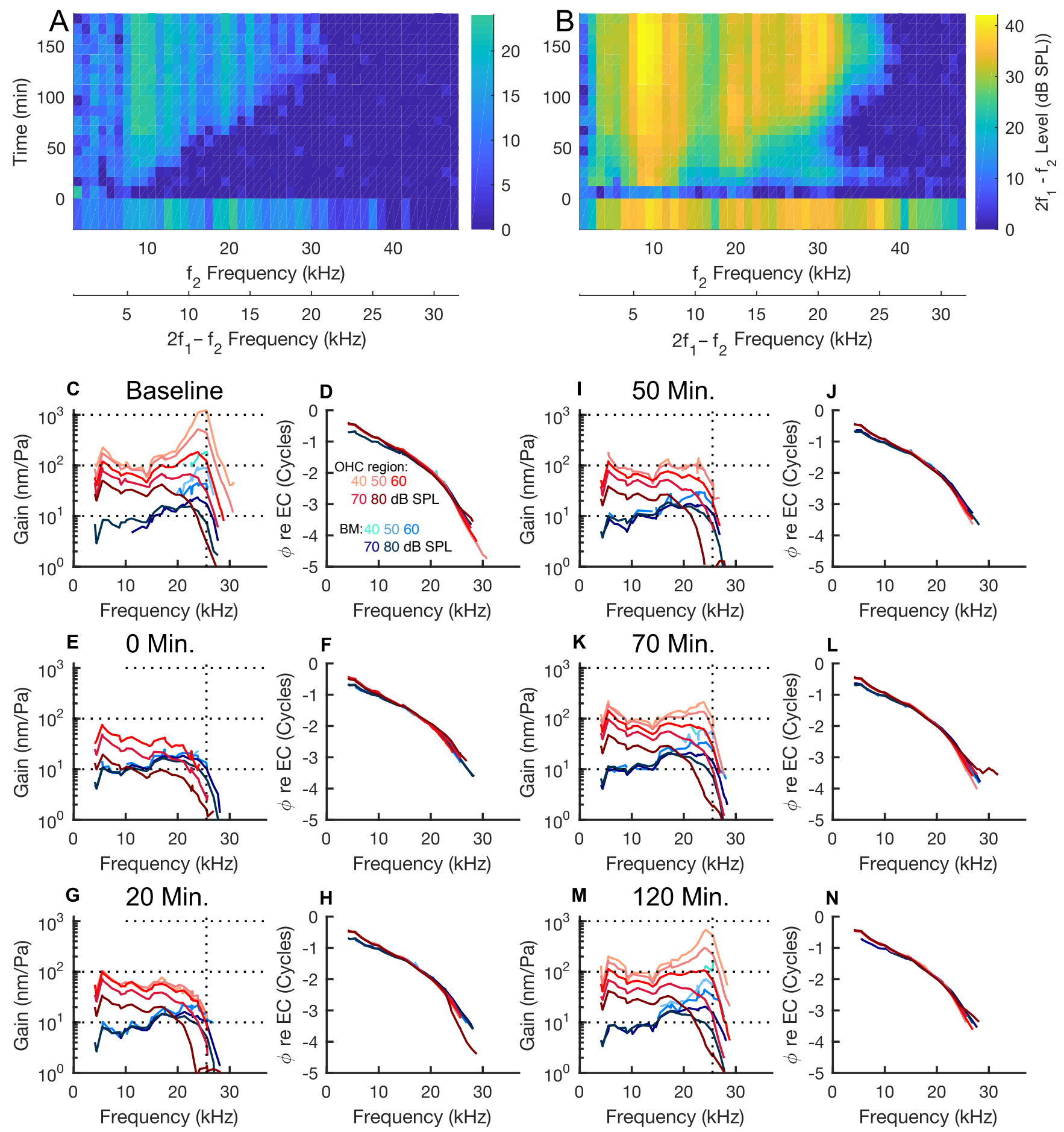

Figure 4: Loss and recovery of amplification following furosemide injection. (A) and (B) show the $2 f_{1}-f_{2}$ DPOAE levels evoked by the $50 \mathrm{~dB}$ SPL (A) and $70 \mathrm{~dB}$ SPL primaries (B). Note the sharp drop immediately following the injection at $t=0$ mins and the gradual recovery which was maximal at $\sim 120$ mins. The two x-axes indicate both the $2 f_{1}-f_{2}$ and the $f_{2}$ frequencies. $(\mathrm{C}-\mathrm{N})$ show the displacements and phases measured at the BM and OHC-regions at different time points. BM / OHC-region vibrations are plotted in blue / red with darker shades indicating higher sound pressure levels; key is in panel D. Vibration amplitude is plotted normalized to the stimulus level in the ear canal, and phase is plotted relative to ear canal pressure. (C) and (D) show the baseline responses, recorded before the injection. (E) and (F) were recorded immediately after and the remaining plots show the results at the indicated time points. By 120 mins, the vibrations had recovered to near baseline levels with no further recovery seen thereafter. Expt. 785, 8-28-2019. 

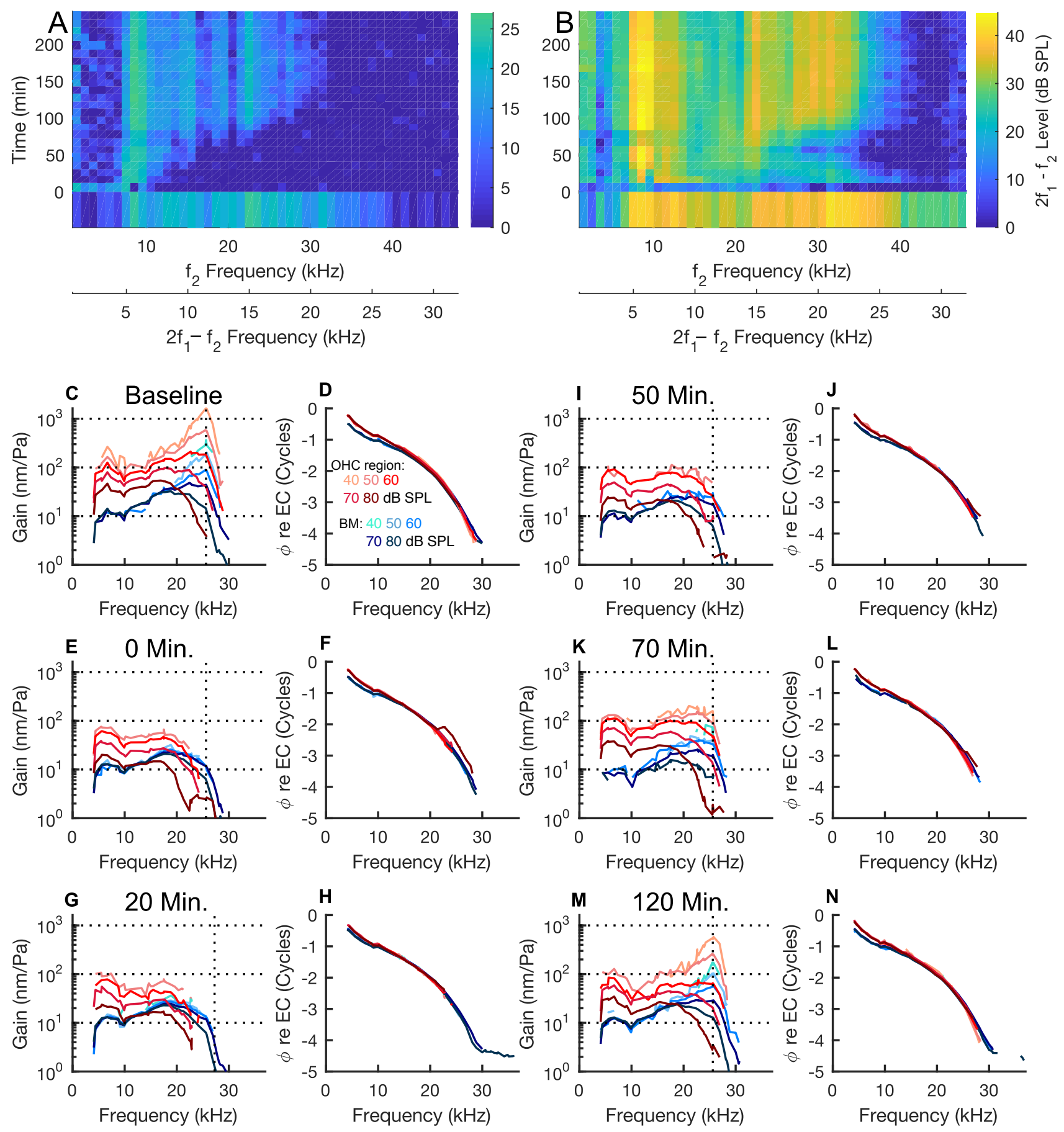

Figure 5: Loss and recovery of amplification in a second cochlea. The layout is as in the previous figure. In this experiment, data were collected for nearly four hours post injection but the recovery of DPOAEs and vibrations reached their fullest extent by about 120 mins post injection and did not recover past that point. Expt. 788, 9-17-19. 
Strimbu et al.
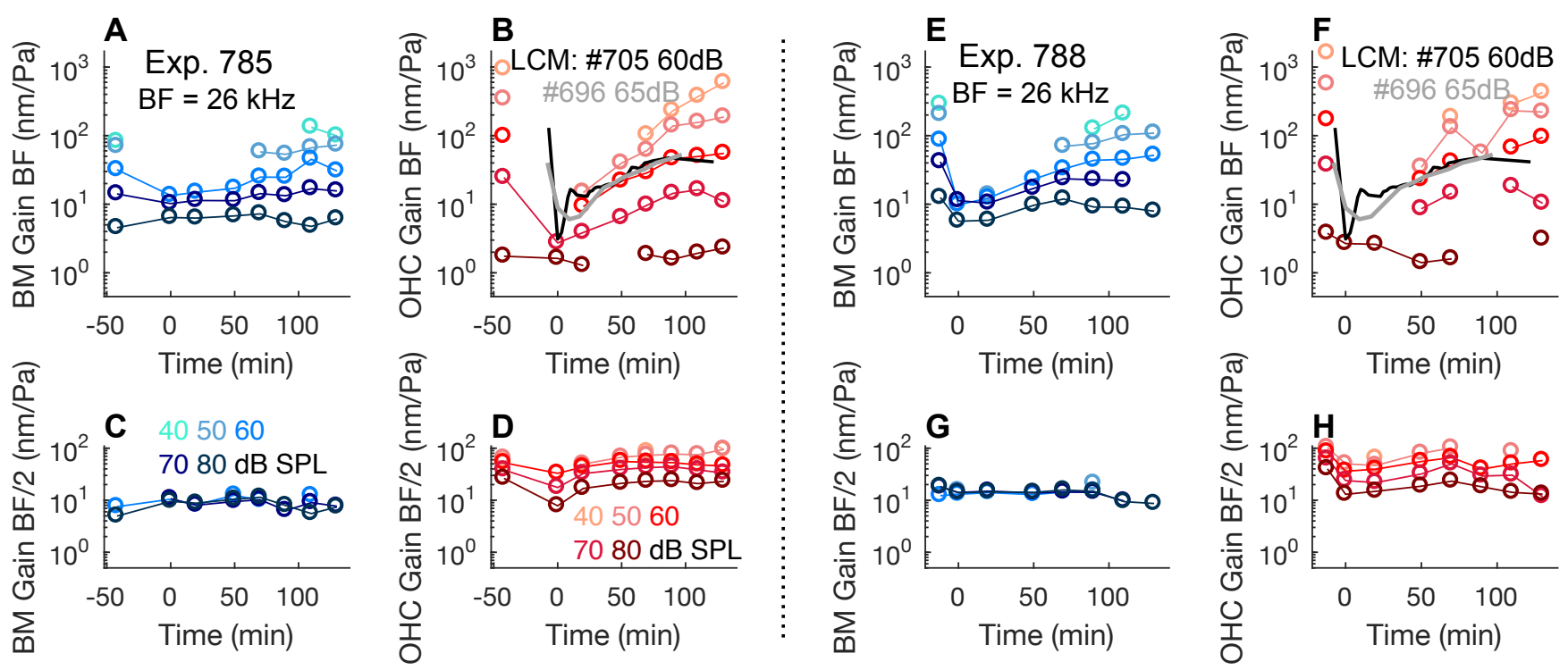

Figure 6: BM and OHC-region vibrations before, after, and during recovery from furosemide treatment, with time $t=0$ right after the injection. Plots on the left are from the same experiment as Fig. 4; those on the right are from Fig. 5. (A) and (E) BM gains at the BF. (B) and (F) OHC-region gains at BF. (C) and (G) BM gains at BF/2. (D) and (H) OHC-region gains at BF/2. Gaps in the data occur when the responses are lower than the noise floor. The relatively long time between baseline and $t=0$ in expt. 785 is present because, as noted in methods, in order to present the fullest (least noisy) baseline measurement data set, the set used for illustration was not always just before the injection. The grayscale data in (B) and (F) are BF LCM results from Fig. 2. These data are included to illustrate that BF LCM and vibration responses recovered on a similar time scale. The $y$-axis for the LCM data have units $\mathrm{mV} / \mathrm{Pa}$, and have been scaled by factors of 60 (expt. 705) and 40 (expt. 696).
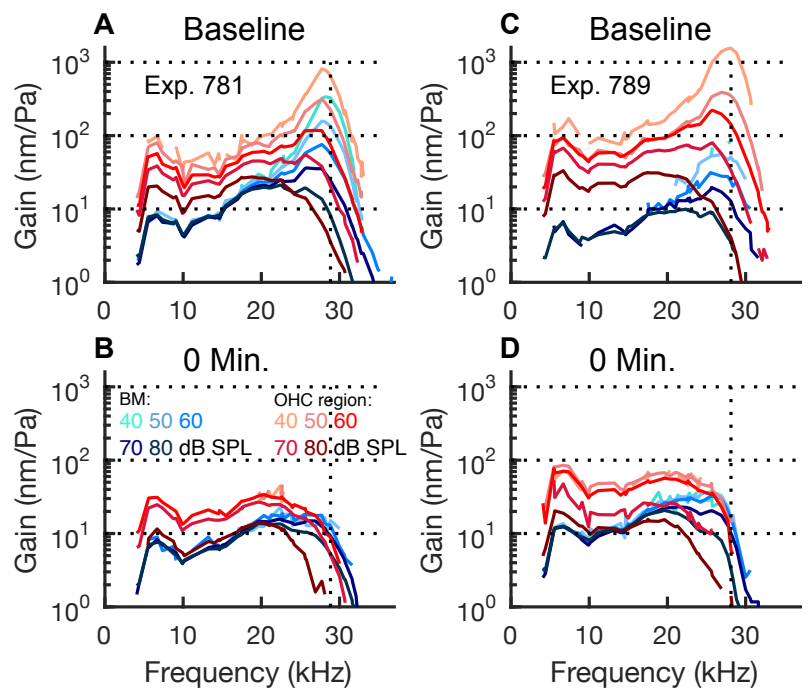

Figure 7: Loss of amplification following furosemide in two preparations that did not recover substantially. Gain relative to stimulus pressure at the ear canal. (A) Baseline, (B) Following furosemide, expt. 781. (C) Baseline, (D) Following furosemide, expt. 789. 


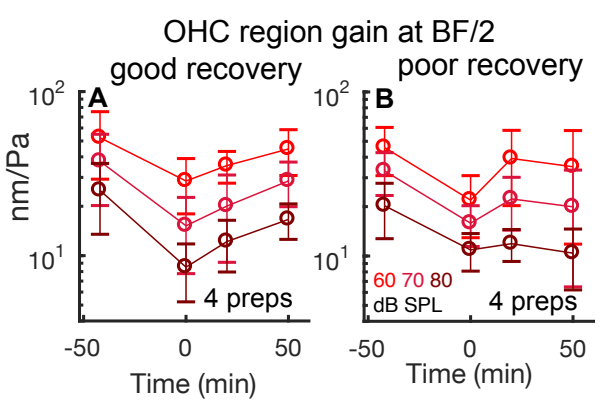

Figure 8: Reduction and recovery of OHC-region sub-BF responses following furosemide. Mean and standard deviation are shown for two groups of preparations. In (A) responses are tracked from the four preparations that showed substantial recovery of cochlear amplification (as in Figs. 4 and 5). In (B) responses are tracked from four preparations in which recovery faltered and never was substantial.
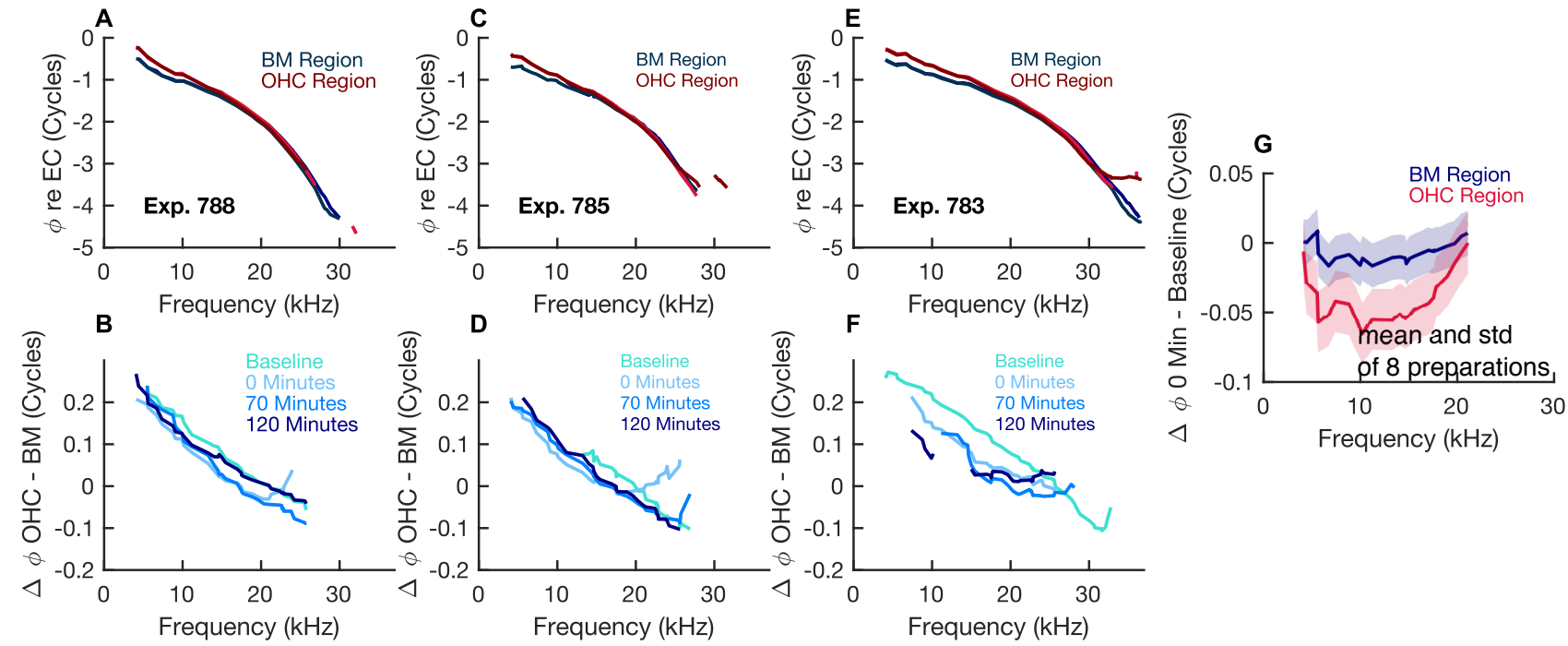

Figure 9: Expanded view of phase differences. Only relatively high SPL results are shown (70 and/or $80 \mathrm{~dB}$ SPL) because at lower SPL data often dropped beneath the noise floor following furosemide. (A, C, E) Subset of baseline phase data from expt.785 (Fig. 4), expt.788 (Fig. 5) and a third (expt.783) that showed substantial recovery. This row reinforces the basic similarity and small but robust differences between $\mathrm{BM}$ and $\mathrm{OHC}$ region phases at baseline. (B, D, F) Phase differences between $\mathrm{OHC}$ region and $\mathrm{BM}$ region, before and just after furosemide $(\mathrm{t}=0)$, at $120 \mathrm{~min}$. when recovery was approximately fullest and at one intermediate time point $(70 \mathrm{~min}) .70 \mathrm{~dB}$ SPL results shown. A three-point smoothing was done on these data to reduce distracting sharp variations. (G) Phase differences within each region before and just after furosemide. Mean and standard deviation from eight preparations are shown. This data is included to show that the BM-region phase changed little, and most of the time-dependent phase differences seen in (B, D, F) resulted from changes in OHC-region phase. $80 \mathrm{~dB}$ SPL results shown. 
Strimbu et al.
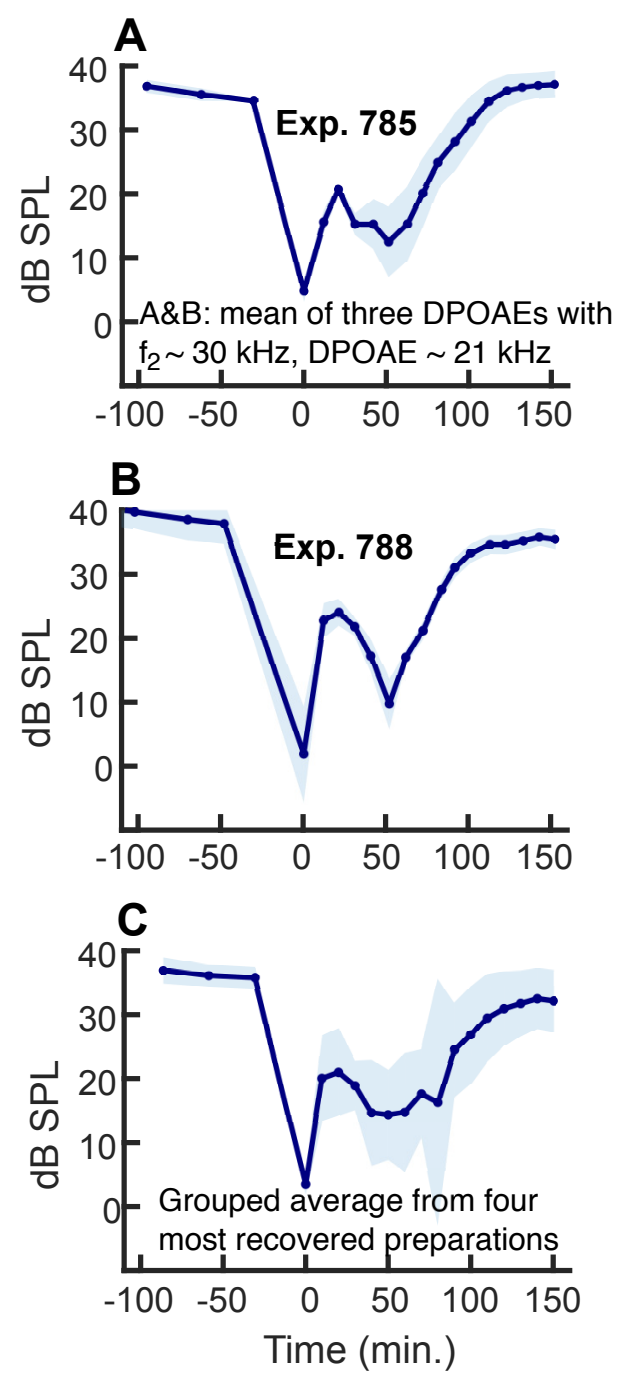

Figure 10: Recovery of DPOAEs. The three DPOAEs around $f_{2}=30 \mathrm{kHz}$, corresponding to DPOAE frequencies $\sim 21 \mathrm{kHz}$ were averaged and mean and standard deviation are shown. Primary levels were $70 \mathrm{~dB}$ SPL. (A) Expt. 785. (B) Expt. 788. (C) Results as in (A) and (B), averaged over the four preparations with substantial recovery. 

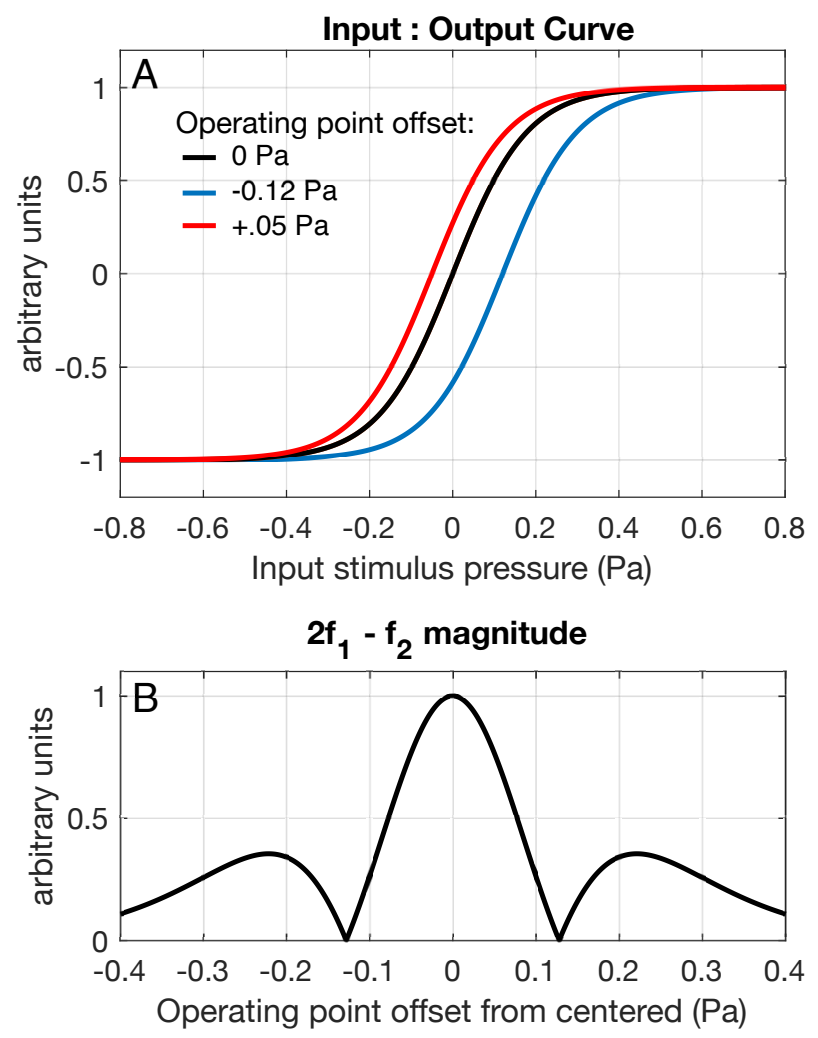

Figure 11: (A) A two-state Boltzmann function representing the nonlinear MET potential $(V)$ as a function of the ear canal pressure $(P)$ and operating point $(O P), V=-V_{\mathrm{sat}}+\left(\frac{2 V_{\mathrm{sat}}}{1+\exp z(P+O P)}\right)$. The slope factor $z=-11.18 \mathrm{~Pa}^{-1}$, was derived in (15) based on LCM responses in the absence of amplification and the function has been plotted here on a normalized scale, with $V_{\text {sat }}=1$. (The nonnormalized value for $V_{\text {sat }}$ was $0.13 \mathrm{mV}$.) The OP shift that occurred after furosemide was shown in Fig. $2 \mathrm{C}$, and extended from -0.12 to +.05 , and these extreme shifts are illustrated here. (B) Using the Boltzmann function, the size of the $2 f_{1}-f_{2}$ generated by this simple nonlinearity is found for a range of operating points. The input SPL of $f_{1}$ and $f_{2}$ was taken as $70 \mathrm{~dB}$ SPL (.06 Pa) to be consistent with Fig. 10. 